\title{
Dissipative soliton resonances in the anomalous dispersion regime
}

\author{
W. Chang, ${ }^{1}$ J. M. Soto-Crespo, ${ }^{2}$ A. Ankiewicz, ${ }^{1}$ and N. Akhmediev ${ }^{1}$ \\ ${ }^{1}$ Optical Sciences Group, Research School of Physical Sciences and Engineering, The Australian National University, \\ Canberra ACT 0200, Australia \\ ${ }^{2}$ Instituto de Óptica, C.S.I.C., Serrano 121, 28006 Madrid, Spain
}

(Received 2 November 2008; published 25 March 2009)

\begin{abstract}
We have numerically calculated the resonance curve and the region of existence of high-energy dissipative solitons in systems governed by the complex cubic-quintic Ginzburg-Landau equation. The calculations are carried out for negative reactive quintic nonlinearity. This choice allows the resonance curve to be continued into the region of positive net dispersion, thus showing that high-energy pulses can also be generated by lasers operating in the anomalous dispersion regime.
\end{abstract}

DOI: 10.1103/PhysRevA.79.033840

PACS number(s): 42.65.Tg, 42.65.Re

\section{INTRODUCTION}

High-energy ultrashort optical pulses are of great importance for a variety of applications [1-3]. The best apparatus for generating them appears to be a passively mode-locked laser system [4,5]. While progress in producing high-energy pulses out of these lasers has been remarkable, most of the results have only been obtained experimentally by using "trial and error" techniques. We have to admit that there is no general theory behind the achievements. The main problem is that each particular laser design requires specific modeling and numerical simulations [6,7]. There is not an "inverse technique" to uncover a system that will produce a specific required output pulse.

However, there is an approach, and it may be considered rather general, which allows us to roughly predict the main properties of pulses generated by these devices. It is based on a single master equation derived using certain approximations [8]. The resulting equation is the complex GinzburgLandau equation or a modification of it. The active nonlinearity in the equation must be at least quintic in order to model the stable generation of pulses. Thus, the simplest model that we can use is the complex cubic-quintic Ginzburg-Landau equation (CGLE). Depending upon the particular device, and the phenomena that we want to describe, the model can be complemented with additional terms in the equation. These could be higher-order dispersion terms, step-wise change in parameters, etc. The additional terms may modify the output pulse and the conditions for its generation but do not destroy the solution completely. If a certain effect is robust, it will persist despite all the modifications.

Specifically, using the CGLE model, the authors of [9] were able to find conditions where the output pulse tends to increase its energy to infinitely large values. This effect was later labeled "dissipative soliton resonance" (DSR) [10]. It turns out that the effect occurs over quite a large region of the system parameters. Basically, there is a codimension-one space of the full space of parameters which admits infinitely large pulse energies. Remarkably, it was found that this subspace is located in the region where dispersion is negative (normal). The fact is in agreement with recent experimental observations of high-energy pulses from passively mode- locked lasers, both fiber [11] and solid-state ones [12]. Indeed, the majority of these observations are in the normaldispersion regime of operation. Thus, the DSR effect has predictive features and, in principle, can be used to design laser systems for the generation of high-energy pulses.

Despite its apparent simplicity, the problem is highly complicated. Even in the simplest model, the number of parameters (six) is beyond easy comprehension so the model requires a significant number of numerical simulations to describe the pulse shape and its parameters. This can take a long time, even using modern computer equipment. Moreover, it is not trivial to make a correspondence between the real experimental parameters and those used in the CGLE model, and so this requires special derivations. While the net cavity dispersion can, in a straightforward manner and after certain normalizations, be represented by the dispersion parameter in the equation, the values of the rest of the coefficients in the equation are complicated functions of those laser parameters which can be adjusted experimentally. This is particularly true for the nonlinear ones.

In this work, we do not attempt to describe any particular laser model. Rather, we consider the DSR effect at a phenomenological level and try to expand our knowledge of the space of parameters where it does exist. In particular, we study the influence of the quintic (reactive) nonlinearity on the resonance. The first question is "can we ignore it?" In other words, can we put $\nu=0$ in Eq. (1) below? For example, if we consider a fiber laser, the cubic reactive nonlinearity comes mainly from the nonlinear susceptibility of the fiber and can be considered to be positive although very small. However, the quintic part of reactive nonlinearity results from the combined effects of the fiber, mode locker, etc., and we cannot, a priori, predict its value. In the case of Kerr-lens mode locking, the net cavity nonlinearity can be an even more complicated function that depends on the cavity design. One thing that is certain is that, for high intensity pulses, even if we do not need it to obtain stable pulses, we cannot ignore it. Hence, in this work, we study the role of the quintic part of the reactive nonlinearity in the DSR.

The second question that we try to answer is: does the DSR effect exist in the anomalous dispersion region? In our previous work [10], we only found the resonance for negative dispersion. On the other hand, there is experimental evidence that high-energy pulses can be generated even when 
there is positive net dispersion in the cavity $[12,13]$. A partial answer to this paradox is also one of the results of the present work.

\section{MODEL}

As in the work [10], we are dealing with the complex cubic-quintic Ginzburg-Landau equation

$i \psi_{z}+\frac{D}{2} \psi_{t t}+|\psi|^{2} \psi+\nu|\psi|^{4} \psi=i \delta \psi+i \epsilon|\psi|^{2} \psi+i \beta \psi_{t t}+i \mu|\psi|^{4} \psi$,

with the same notation as before. Active, or dissipative, terms are on the right-hand side of the equation while all reactive terms are on the left. The coefficients $\delta, \epsilon$, and $\mu$ are mainly determined by the gain in the system, cavity losses, and transmission characteristics of the mode-locking device while $\beta$ roughly describes the main part of the spectral response of the cavity. On the left-hand side, $D$ is responsible for the net dispersion in the cavity while $\nu$ describes the active part of the reactive nonlinearity. The cubic part is assumed to be positive, normalized, and taken to be equal to one. As mentioned, the model is purely phenomenological. For each particular laser, the coefficients can be calculated in a similar fashion to the case of the fiber laser in [7]. The coefficients here could be very complicated functions of the real laser parameters and specific design factors. However, their exact representations are not really needed for understanding the laser operation at an intuitive level.

Finding sets of parameters for which the CGLE has soliton solutions is a tedious task which has been carried out in a few previous works $[14,15]$. This set is not necessarily a single region. There could be several sets, with each one having distinct soliton properties [16]. The regions can be adjacent to each other [15] or separated in the space of parameters [16]. The structure of the regions is extremely complicated and it is hard to imagine that all of them will ever be known. However, the solutions with the most remarkable features can be distinguished, of course. In particular, we found a region of parameters of the CGLE which admits solitons with extraordinarily high energy. We called this effect dissipative soliton resonance [10].

We solved Eq. (1) using a split-step technique with fast Fourier transform of the pulse applied for linear terms in the equation and iterations for nonlinear terms. Stable stationary solutions are found as a result of the convergence of localized initial conditions to them. As stable solutions serve as attractors in dissipative dynamical systems, they can be found with relative ease, provided the initial conditions are reasonably close to them. To accelerate the convergence, we used solutions found for a certain set of parameters as initial conditions for the next-nearest point in the parameter space. This technique works well for points far from the resonances. Then, the pulses at the nearby points are similar to each other. When parameters are close to the resonance, the pulse shapes change quickly with a change in parameters. In that range, on moving from point to point, we have to change step sizes, numerical windows, and the number of points in

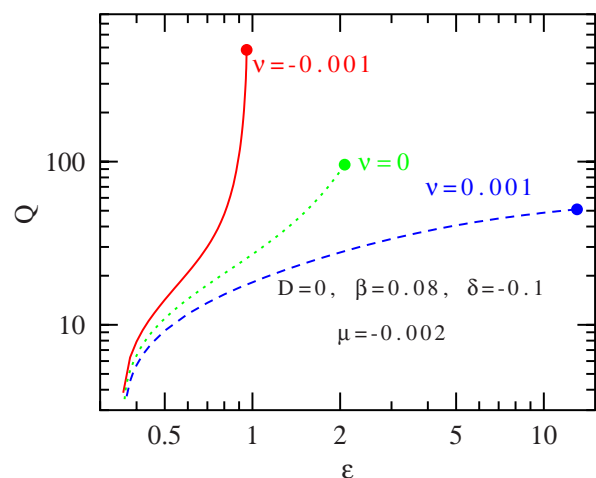

FIG. 1. (Color online) Energy, $Q$, of the pulse versus $\epsilon$ for negative (red solid line), zero (green dotted line) and positive (blue dashed line) values of $\nu$. The curve for negative $\nu$ shows the resonant increase in energy, $Q$.

the numerical grid. These procedures made the method more time consuming but technically it is still relatively simple. The only difficulty lies in the higher inaccuracies when the soliton energy becomes high. At some point, we had to stop further calculations, but of course this does not mean that solitons with even higher energies cannot exist.

\section{RESULTS}

The role of the higher-order reactive nonlinearity term, $\nu$, in the model requires special attention. Hence, first, we studied the dependence of the soliton energy on $\nu$. Curves of the soliton energy, $Q$, versus $\epsilon$ for three values of $\nu$ are shown in Fig. 1 on a log-log scale. The curves end at those values of $\epsilon$ where no stable solution is found. In these studies, we took the net dispersion, $D$, to be zero as a transitional value between normal and anomalous dispersions. In fact, if we find the resonance phenomenon for $D=0$, then it is very likely that DSR will also exist in the anomalous dispersion region. Indeed, we found that changing $\nu$ from a positive to a negative value increases the maximum reachable soliton energy and transforms the $Q(\epsilon)$ dependence into a resonance-like curve. This can be clearly seen in Fig. 1. The curve for $\nu=-0.001$ has the fastest energy increase in a small region of $\epsilon$ while the curve for $\nu=+0.001$ shows a very slow increase. To show this difference clearly, the scale for $\epsilon$ was also chosen to be logarithmic. The curves for more negative values of $\nu$ show an even quicker growth of the energy with $\epsilon$.

The pulse profiles and their spectra at the points on these curves with the highest energy, indicated by thick dots, are shown in Fig. 2. The pulses for positive $\nu$ are narrower and higher than those for negative $\nu$. In the case of positive $\nu$, as the cubic gain $(\epsilon)$ increases, the pulses become narrower and of higher intensity. The opposite happens for negative $\nu$, where the increase in energy as $\epsilon$ increases is mainly due to the increase in the pulse width. This is particularly true as we get closer to the resonance.

These observations mean that the region of existence of high-energy solitons must be constructed for the case of negative $\nu$. Although the value, and even the sign, of $\nu$ for laser systems are usually unknown, the comparison of pulse 

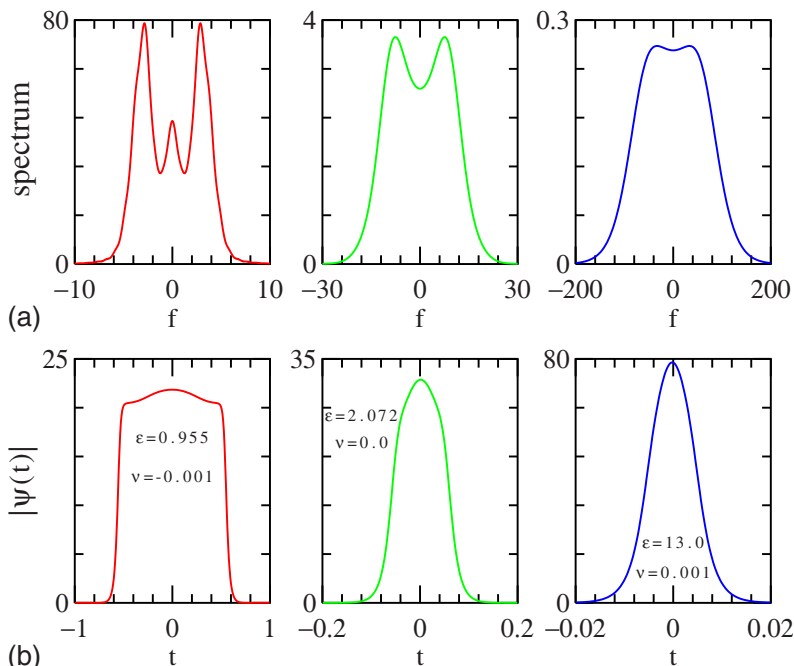

FIG. 2. (Color online) (a) Soliton spectra and (b) pulse profiles of the solutions approaching the resonance. The common system parameters are $D=0, \delta=-0.1, \beta=0.08$, and $\mu=-0.002$. The values of $\epsilon$ and $\nu$ for each case are written in the figure for the pulse amplitude (bottom row). The three cases correspond to the three solid dots shown in Fig. 1.

shapes and energies at the qualitative level could give an indication of it. The results of extensive numerical simulations are shown in Fig. 3. A significant amount of computational time was required to obtain each point in the plot. It is a contour plot of the region in $(\epsilon, D)$ domain showing lines of equal energy, $Q$. (In the online version of the paper, the contours are shown in color. The highest energies are shown

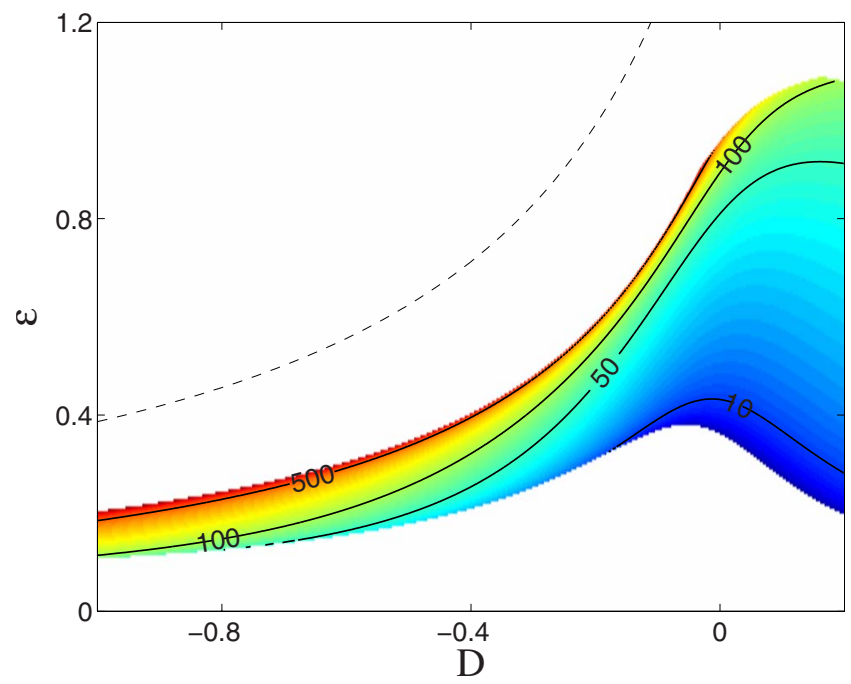

FIG. 3. (Color online) Region of existence of stable solitons in $\epsilon-D$ plane. Color indicates the soliton energy which is highest at the upper boundary of the region (red). The contour lines drawn inside this region correspond to fixed values of the soliton energy with the highest value, $Q=500$, and the lowest value, $Q=10$. The rest of the system parameters are fixed at $\delta=-0.1, \beta=0.08, \nu=-0.001$, and $\mu=-0.002$. The dashed line plotted above the region of existence is the resonance curve found using a simple approximation based on a trial function [10]. Despite the rough approximation used, the curve qualitatively describes the resonance quite well. in red). The left upper edge of the region corresponds to the highest energies found in the numerical simulations. This upper edge extends to the region with zero and even positive dispersion, $D$, in contrast to the case found for positive $\nu$ [10]. Several black contour lines shown inside the region of existence correspond to selected values of energy. To be specific, the energy levels are $Q=10,50,100$ and 500. Naturally, the line $Q=500$ is the closest to the upper edge of the region, which is the resonance curve. Solitons have the smallest energy, $Q$, at the lower edge of the region. Outside the region of existence (white areas) stable stationary solitons do not exist.

An analytic approximate expression for the resonance curve, in terms of the system parameters, has been found previously in [10]. Thus, using the generalized Gaussian trial function

$$
\psi(t, z)=A \exp \left(-\frac{t^{2}}{w^{2}}-\frac{t^{4}}{w^{4}}\right) \exp \left(i c t^{2}\right),
$$

where $A$ is the soliton amplitude, $w$ is the soliton width, and $c$ is the soliton chirp, we obtained

$$
D=\frac{0.25 k \beta(1.319 k \nu-3.410 \mu)}{\mu(0.135 k \epsilon-1.793 \delta \mu)},
$$

where

$$
k=1.588 \epsilon+\sqrt{2.522 \epsilon^{2}-9.632 \delta \mu} .
$$

Equation (3) relates the parameters of the CGLE which basically provide the highest energy soliton. At any twodimensional projection of the complete parameter space, this equation can be represented as a curve. This curve is shown in Fig. 3 by the black dashed line above the region of existence. As trial function (2) is not a real solution of Eq. (1), the curve is located at an appreciable distance from the resonance curve found numerically. However, it is very much "parallel" to the upper boundary of the region of soliton existence, thus showing good qualitative agreement with the numerical simulations. We cannot expect quantitative agreement due to the roughness of approximation (2). Moreover, when the dispersion, $D$, becomes positive, the agreement is worse. It is likely that trial function (2) should be changed in this region to have a better fit.

Figure 3 shows that the resonance effect is more pronounced in the negative dispersion region. When moving to the right-hand side of the region, the values of soliton energy are lower. Nevertheless, the resonance does exist in the sense that the energy goes up when the parameters reach the resonance curve in the upper part of the region. In Fig. 4, we show the curve for soliton energy, $Q$, versus $D$ in the vicinity of the resonance when the dispersion, $D$, is positive. We have chosen the value $\epsilon=1.0$, which is near the upper limit of the region of existence in Fig. 3. The curves for other values of $\epsilon$ near $\epsilon=1.0$ are similar to this one. The energy has a clear tendency to increase, as can be seen from the figure. The maximum shown in this figure is defined by the limitations of the numerical simulations rather than by the actual energy of the soliton. Thus, the resonance evidently does exist in the positive dispersion regime. The correct choice of system pa- 


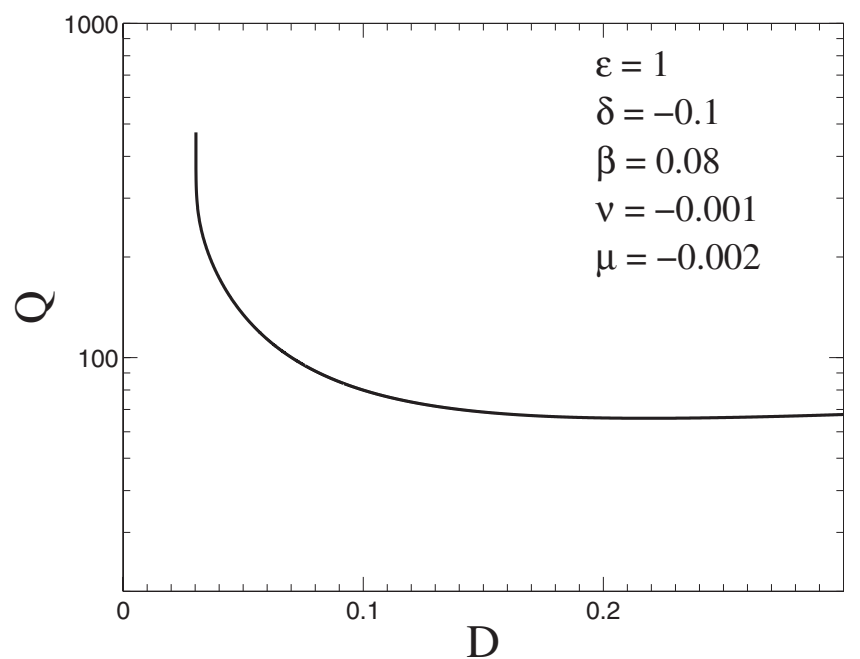

FIG. 4. Dissipative soliton resonance effect in the anomalous dispersion regime. The energy of the soliton increases quickly near $D \approx 0.03$, which is the resonance point for the given set of parameters shown inside the plot. The energy curve shown here has an upper limit due to the inaccuracy of numerical simulations above this point.

rameters, and $\nu$ in particular, is a necessary condition for its appearance. These observations are in line with experimental results $[12,13]$ of high-energy solitons in the case of net positive dispersion in the cavity.

The pulse shapes and their profiles, for the same set of parameters, are shown in Fig. 5. Three consecutive examples for $D=0.1,0.05$ and 0.0305 are given. When approaching the resonance, the pulse profile widens and assumes a nearly rectangular shape. The limit of $D=0.0305$ is again defined by the limitations of numerical simulations. The spectra increase their amplitudes and reveal two side maxima. This latter feature has been observed in most of the experiments dealing with the generation of high-energy pulses [11,17-20]. The pulses are highly chirped, as in previous studies $[19,20]$. Dechirping allows us to compress them up to their transform limits by using dispersion compensation lines [21-23]. Numerical examples of pulse compression for solitons near the resonance curve have been presented earlier in [24].

\section{DISCUSSION}

Originally, it was noted that solitons of the nonlinear Schrödinger equation (conservative bright solitons) physically existed due to the balance between dispersion and reactive nonlinearity [14]. For positive cubic nonlinearity, this balance required the dispersion to be positive. When extending the notion of solitons to dissipative systems, we have to admit that there must be a complex balance between dispersion, nonlinearity, gain and loss. For stationary solutions, the balance between gain and loss has to be exact. This allows the dispersion to be shifted into the negative region, thus lifting the original balance in favor of the balance between gain and loss. Moreover, it turns out that solitons in the negative dispersion region can reach higher energies than those in the positive dispersion case. This observation leads to impor-
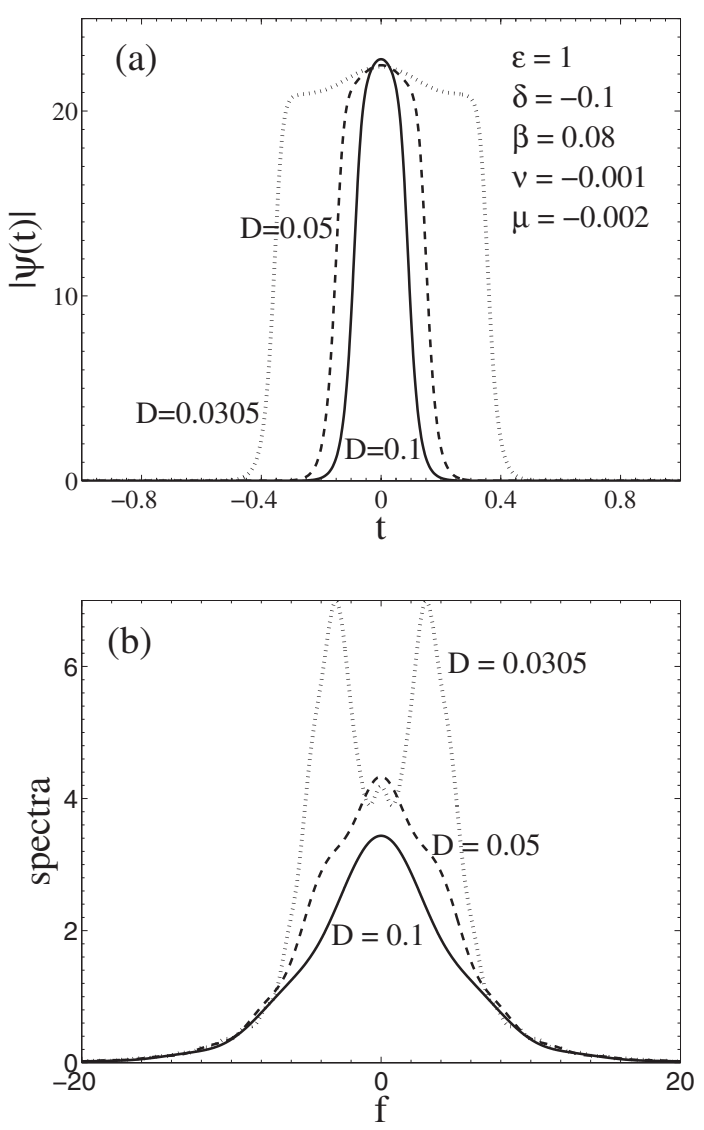

FIG. 5. (a) Soliton profiles and (b) their spectra when the solutions approach the resonance. The system parameters are $\epsilon=1$, $\delta=-0.1, \beta=0.08, \nu=-0.001$, and $\mu=-0.002$.

tant applications in the design of passively mode-locked lasers with net negative dispersion [11].

On the other hand, the master equation that describes laser systems has many parameters. It is not an easy task to study the influence of all of them on the soliton properties. Our present calculations were performed for the case of negative quintic reactive nonlinearity. This choice allows for the resonance curve for dissipative solitons to be continued into the region with positive dispersion, thus showing that highenergy pulses can be generated by lasers operating in the anomalous dispersion regime. Clearly, more studies are needed in order to optimize the parameters of the system that generates pulses with the highest possible energies.

In conclusion, we have numerically calculated the resonance curve and regions of existence of high-energy dissipative solitons in systems governed by the complex cubicquintic GLE. Our results can be useful for developing laser systems with passive mode locking that can generate highenergy short pulses.

\section{ACKNOWLEDGMENTS}

The work is supported by the Australian Research Council (Discovery Project Scheme Contract No. DP0663216). J.M.S.C. acknowledges support from the M.E.y C. under Contract No. FIS2006-03376. 
[1] I. P. Christov, M. M. Murnane, and H. C. Kapteyn, Phys. Rev. Lett. 78, 1251 (1997).

[2] G. Genty, S. Coen, and J. M. Dudley, J. Opt. Soc. Am. B 24, 1771 (2007).

[3] I. Maxwell, S. Chung, and E. Mazur, Med. Laser Appl. 20, 193 (2005).

[4] A. Chong, J. Buckley, W. H. Renninger, and F. W. Wise, Opt. Express 14, 10095 (2006).

[5] B. Ortac, O. Schmidt, T. Schreiber, J. Limpert, A. Tünnermann, and A. Hideur, Opt. Express 15, 10725 (2007).

[6] W. H. Renninger, A. Chong, and F. W. Wise, Phys. Rev. A 77, 023814 (2008).

[7] A. Komarov, H. Leblond, and F. Sanchez, Phys. Rev. E 72, 025604(R) (2005).

[8] H. A. Haus, IEEE J. Quantum Electron. 11, 736 (1975).

[9] N. Akhmediev, J. M. Soto-Crespo, and Ph. Grelu, Phys. Lett. A 372, 3124 (2008).

[10] W. Chang, A. Ankiewicz, J. M. Soto-Crespo, and N. Akhmediev, Phys. Rev. A 78, 023830 (2008).

[11] A. Chong, W. H. Renninger, and F. W. Wise, Opt. Lett. 32, 2408 (2007)

[12] S. H. Cho, F. X. Kärtner, U. Morgner, E. P. Ippen, J. G. Fujimoto, J. E. Cunningham, and W. H. Knox, Opt. Lett. 26, $560(2001)$
[13] B. Ortaç, M. Plötner, T. Schreiber, J. Limpert, and A. Tünnermann, Opt. Express 15, 15595 (2007).

[14] N. Akhmediev and A. Ankiewicz, Solitons, Nonlinear Pulses and Beams (Chapman and Hall, London, 1997).

[15] N. Akhmediev, J. M. Soto-Crespo, and G. Town, Phys. Rev. E 63, 056602 (2001).

[16] A. Ankiewicz, N. Devine, N. Akhmediev, and J. M. SotoCrespo, Phys. Lett. A 370, 454 (2007).

[17] A. Fernandez, T. Fuji, A. Poppe, A. Furbach, F. Krausz, and A. Apolonski, Opt. Lett. 29, 1366 (2004).

[18] V. L. Kalashnikov, E. Podivilov, A. Chernykh, S. Naumov, A. Fernandez, R. Graf, and A. Apolonski, New J. Phys. 7, 217 (2005).

[19] V. L. Kalashnikov, E. Podivilov, A. Chernykh, and A. Apolonski, Appl. Phys. B: Lasers Opt. 83, 503 (2006).

[20] S. Naumov, A. Fernandez, R. Graf, P. Dombi, F. Krausz, and A. Apolonski, New J. Phys. 7, 216 (2005).

[21] E. B. Treacy, IEEE J. Quantum Electron. 5, 454 (1969).

[22] A. M. Weiner, Prog. Quantum Electron. 19, 161 (1995).

[23] B. J. Eggleton, G. Lenz, and N. M. Litchinitser, Fiber Integr. Opt. 19, 383 (2000).

[24] W. Chang, A. Ankiewicz, J. M. Soto-Crespo, and N. Akhmediev, J. Opt. Soc. Am. B 25, 1972 (2008). 